them good, especially the drawings of instruments and diagrams of operations. The style is such that one can read on without getting readily tired; some of it may even be called light reading, as witness the passage already quoted on the methods of supporting the perineum.

The great value of the work is its practicalness. Little points of detail turn up on almost every page, showing that it is, like Dr. Percival Willughby's book on Midwifery, " not feigned, or the surmised thoughts, nuctors, or man's fantasie, sitting and meditating in his studye," but the work of a man who has often done what he wishes to teach his reader to do.

We cannot but commend the book to those who are, or who wish to become, gynecologists.

The Pharmacopoia of the British Hospital for Diseases of the Skin. Edited by Balmanno SQUIRE, M.B. Lond. London : J. \& A. Churchill. 1880.

THE editor's aim in arranging this collection of remedies has been "to attain simplicity in the formulæ and conciseness in the directions," and in this endeavour he has been almost too successful, as in many instances the simplicity and conciseness of his prescriptions degenerate into mere baldness and inelegance, such as one hardly looks for from any one bearing the name of Squire. Thus, the "mixtures," to a large extent, consist simply of the average dose of the active ingredient dissolved in an ounce of water; the use of adjuvants, corrigents, \&c., being neglected in a manner that would have been condemned by many, especially of the older authors. It is to be regretted also that the work should present such an incomplete guide to the practice at the British Hospital for Skin Diseases, as must necessarily be the case from the fact that all prescriptions for skin treatment already provided in the British Pharmacopœia are omitted. The practitioner, therefore, who expects to find in this book, as its title might suggest, a moderately exhaustive grouping of all the approved formulæ used in dermatological practice, together with intelligible directions as to the circumstances and manner in which the remedies are to be employed, will be disappointed; for this he must still trust to his text book of skin diseases. Apart from these drawbacks, however, the work is one of value. Even the mere reading over of this list of prescriptions could scarcely fail to suggest some line of treatment to those in difficulties, particularly as most of the newer additions to skin therapeutics are here noticed. The means of reference 
are ample, the index taking up 16 of the 92 pages of which the work consists. The prescriptions are also so constructed as to be easily remembered, the total quantity of each being reduced, whenever possible, to one ounce. There are several small slips of the pen in these pages which would bear correction, thus:- huile de cade is not obtained from Juniperus Communis, but from J. Oxycedrus; pilula podophylli cum nuce vomicae is bad Latin; the direction to wash with "warm soap and water" is clumsy; and the statement that Venetian talc is a mineral "of an unctuous feel" is inelegant.

Atlas of Skin Diseases. Part VII. By Dr. LouIs A. Duhring. Philadelphia: J. B. Lippincott \& Co. 1880.

Some months ago we had occasion to notice parts I to VI of this excellent Atlas, and expressed a very favourable opinion regarding them. This part quite sustains the high reputation established by its predecessors. It includes lithographic illustrations of eczema pustulosum, impetigo contagiosa, syphiloderma papulosum, and lupus vulgaris. The representations given by these plates of the various diseases mentioned are most faithful and life-like, and approach as near perfection as anything of the kind we have seen. The accompanying text is, as before, concise, practical, and clear; it consists essentially of a description of the particular case chosen for illustration, and the method of treatment adopted, together with a few more general observations on the nature and course of the affection. We again commend this Atlas to the attention of practitioners and others.

\section{PERISCOPE OF DERMATOLOGY.}

No. II.

By A. NAPIER, M.D.

Physician for Skin Diseases, Anderson's College Dispensary.

1. Atropine Eczema.-In this case the introduction of a few drops of a 1 per cent solution of sulphate of atropine into the eye was invariably followed by severe eczema and pseudo-erysipelatous swelling and redness of the face and neck. The patient was a woman of forty-five, of good con- 